Document downloaded from:

http://hdl.handle.net/10251/54637

This paper must be cited as:

Gozálvez Serrano, D.; Gómez Barquero, D.; Vargas Paredero, DE.; Cardona Marcet, N. (2013). Combined time, frequency and space diversity in DVB-NGH. IEEE Transactions on Broadcasting. 59(4):674-684. doi:10.1109/TBC.2013.2281665.

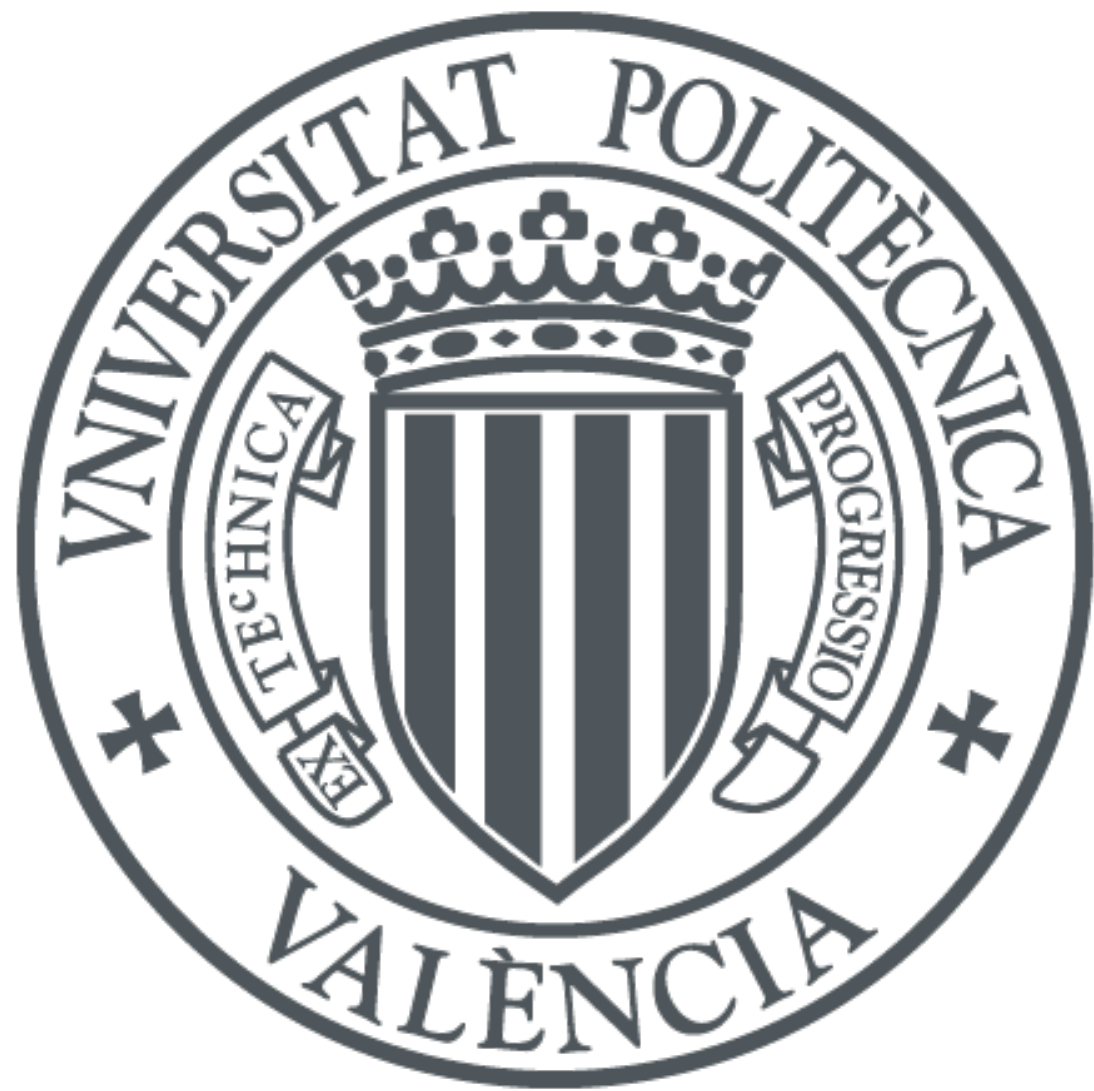

The final publication is available at

http://dx.doi.org/10.1109/TBC.2013.2281665

Copyright Institute of Electrical and Electronics Engineers (IEEE)

Additional Information

"(C) 2013 IEEE. Personal use of this material is permitted. Permission from IEEE must be obtained for all other uses, in any current or future media, including reprinting/republishing this material for advertising or promotional purposes, creating new collective works, for resale or redistribution to servers or lists, or reuse of any copyrighted component of this work in other works." 


\title{
Combined Time, Frequency and Space Diversity in DVB-NGH
}

\author{
David Gozálvez, David Gómez-Barquero, David Vargas, and Narcís Cardona \\ Instituto de Telecomunicaciones y Aplicaciones Multimedia (iTEAM) \\ Universitat Politècnica de València (UPV) \\ Email: \{dagoser, dagobar, davarpa, ncardona\}@iteam.upv.es
}

\begin{abstract}
In this paper we investigate the combined use of time, frequency, and space diversity in DVB-NGH, the next generation mobile broadcasting standard. Compared to current standards like DVB-H or DVB-SH, the largest improvement in next generation systems is expected to be achieved by means of better diversity in the time, frequency, and space domains. In this sense, DVB-NGH is the first broadcasting system to exploit the use of diversity in the three domains by incorporating at the physical layer long time interleaving (TI), time-frequency slicing (TFS), and multiple-input multiple-output (MIMO). This paper investigates the gains of using the three types of diversity in DVB-NGH, as well as the mutual influence between different types of diversity. To this purpose, we employ an informationtheoretic approach based on the outage capacity of the channel in addition to physical layer simulations.
\end{abstract}

Index Terms-Diversity, DVB-H, DVB-NGH, DVB-SH, time interleaving, multiple-input multiple-output, time-frequency slicing.

\section{INTRODUCTION}

From a commercial perspective, the next generation of mobile broadcasting systems should be designed to provide better coverage and robustness. Networks aimed at wide coverage area should be efficient and affordable to build, while the transmitted signal should be robust enough to enable reception stationary, walking, or moving at high speeds [1]. The adoption of turbo codes and low-density parity check (LDPC) codes in second generation systems such as DVB-T2 (Digital Video Broadcasting - Terrestrial 2nd Generation), already achieves a capacity within $1 \mathrm{~dB}$ of the Shannon limit in AWGN [2], and thus, major gains cannot be expected by means of improved forward error correction (FEC). Therefore, the use of diversity in the time, frequency, and space domains is necessary in the next generation of mobile broadcasting systems to achieve a significant performance increase compared to the current generation. In this sense, DVB-NGH (Next Generation Handheld) will be the first broadcasting system to include the use of diversity in the three domains by incorporating at the physical layer long time interleaving (TI), time-frequency slicing (TFS), and multiple-input multiple-output (MIMO) techniques. The combined use of time, frequency, and space diversity will provide DVB-NGH with superior robustness and better coverage than existing mobile broadcasting standards, including DVB-H [3] and DVB-SH [4]. The standard includes terrestrial profiles and also hybrid profiles that combine terrestrial and satellite transmissions. The standardization process of
DVB-NGH is currently at its final stages and it is expected to be completed by September of 2012 .

Mobile broadcasting systems generally incorporate time diversity across hundreds of milliseconds, frequency diversity inside a single RF channel, and space diversity with multiple receive antennas (single-input multiple-output, SIMO). Long TI exploits the time diversity of the mobile channel across several seconds so that it is possible to cope with the presence of long error bursts in the received signal. Together with long TI, fast zapping is needed in order to maintain the zapping time within tolerable values (i.e. below 2 s). The utilization of long TI is generally considered as key for land mobile satellite (LMS) reception [5], while it can also provide very important gains for terrestrial transmissions in high-speed reception scenarios (vehicles, trains) [6]. Upper layer - forward error correction (UL-FEC) techniques are generally proposed for long TI due to the fact that they can provide long interleaving durations with more relaxed hardware requirements. In contrast, the implementation of long $\mathrm{TI}$ at the physical layer provides the most effective protection as it employs all the available soft-information from the channel [7].

TFS has been included in DVB-NGH to increase the transmission robustness by means of improved frequency diversity [8]. Mobile broadcasting systems based on orthogonal frequency-division multiplexing (OFDM) generally achieve frequency diversity by interleaving the FEC codewords inside a single RF channel (e.g. $8 \mathrm{MHz}$ ). While this achieves sufficient robustness against multipath propagation in the UHF band, it cannot compensate for the signal variations encountered between different RF channels [9]. The incorporation of TFS in DVB-NGH offers potentially better diversity by extending the interleaving up to hundreds of $\mathrm{MHz}$ in the frequency domain.

One of the largest improvements in the next generation of mobile broadcasting systems is expected to come from the incorporation of MIMO techniques [10]. The implementation of multiple antennas in both ends of the communication channel is twofold; on one hand, it improves the reception robustness by means of better diversity, on the other hand, it provides higher capacity by exploiting the multiplexing capabilities of the MIMO channel [11]. The implementation of MIMO in DVB-NGH is accomplished by means of cross-polarized antennas. These are better suited to handheld reception in the UHF band, since the implementation of spatially separated 


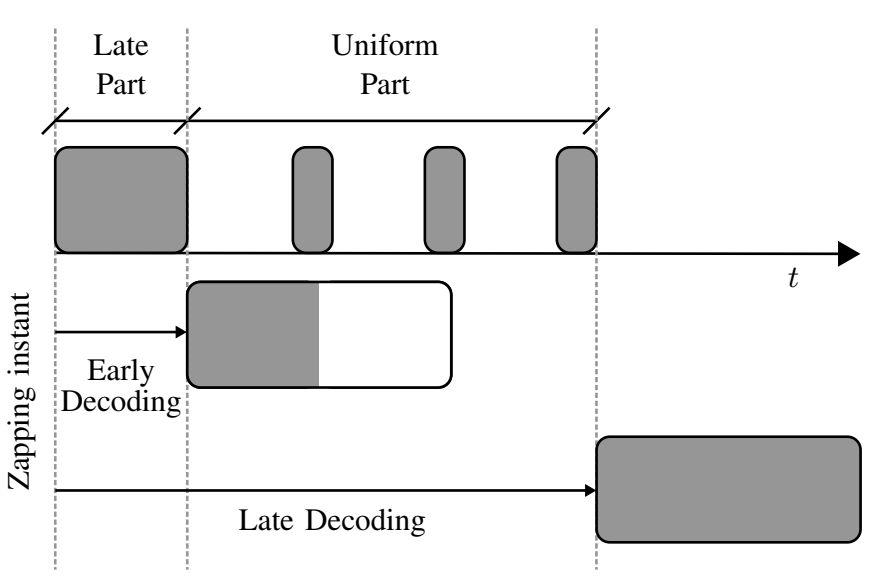

Fig. 1. Comparison between early and late decoding with uniform-late interleaving. Early decoding is performed after the reception of the late part, whereas late decoding is performed after receiving all the data and parity information in the late and the uniform part.

antennas in handheld devices is generally impractical at UHF frequencies due to the required separation between antennas (e.g. $15 \mathrm{~cm}$ at $600 \mathrm{MHz}$ ).

In this paper, we investigate the combined use of time, frequency, and space diversity in DVB-NGH, the performance gains that can be achieved in mobile channels, and the mutual influence between the different types of diversity. In previous investigations, the utilization of time, frequency, and space diversity in mobile broadcasting systems has been studied in a separate manner, and a joint investigation like the one presented in this paper has not been performed. Besides DVB-NGH physical layer simulations, we employ an information-theoretic approach based on the outage capacity of the channel to evaluate the potential gains of diversity techniques [12], [13], and to compare the simulated performance of DVB-NGH with the theoretical maximum.

The rest of the paper is organized as follows. In Section II, we describe the utilization of long TI, TFS, and MIMO in the DVB-NGH standard. In Section III, we show the potential gains of diversity techniques based on the outage capacity of the channel. In Section IV, we present the simulation results that illustrate the improvement achieved by diversity techniques in DVB-NGH systems. Finally, we give some concluding remarks in Section V.

\section{Diversity TeChNiQUES IN DVB-NGH}

\section{A. Long Time Interleaving}

DVB-NGH adopts a combination of intra-frame block interleaving (BI) and inter-frame convolutional interleaving (CI) for the provision of TI at the physical layer. The BI extends across hundreds of milliseconds in order to accomplish a uniform interleaving of information symbols within the frames. The CI extends up to several seconds by distributing the information symbols of each FEC codeword across multiple frames. Convolutional interleavers possess some important advantages in terms of latency and memory utilization that makes them well suited for inter-frame interleaving. In particular, the endto-end latency and the memory requirements of convolutional

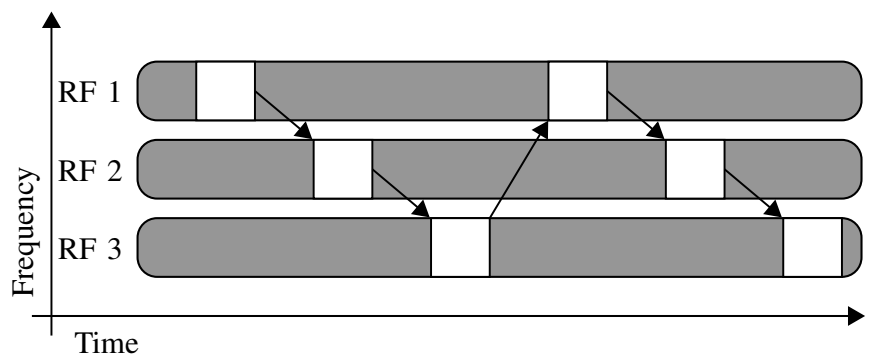

Fig. 2. Time-frequency slicing performed across three RF channels. The time slices are transmitted sequentially in the channels that constitute the TFS multiplex.

interleavers are approximately half as large as with block interleavers.

Broadly speaking, the performance of long TI can be divided in early decoding and late decoding. The first case enables fast zapping and refers to the possibility of performing channel switching prior to the reception of all the data and parity information. The second case provides the highest robustness and refers to the decoding of codewords when all the information is available at the receiver side. In order to improve early decoding and facilitate fast zapping, the hybrid terrestrial-satellite profiles of DVB-NGH support uniform-late configurations of $\mathrm{CI}$ similar to the uniform-late interleaving profiles included in DVB-SH [14]. As it is shown in Fig 1, a large portion of information from the codewords is transmitted in a late part with a duration of typically hundreds of milliseconds, so that the receiver can start decoding the service immediately after the reception of the late frame. However, this results in a non-uniform interleaving of information over time, which might reduce the reception robustness of late decoding in mobile scenarios. The trade-off between early and late decoding is therefore determined by the percentage of information that is carried in the late part. Uniform-late configurations with more than $50 \%$ of information in the late part are generally not practical due to the performance degradation in mobile channels.

\section{B. Time-Frequency Slicing}

Traditionally, broadcasting services are grouped into multiplexes before being transmitted over the air, with each multiplex being allocated to one RF channel. When TFS is used, the services are multiplexed across several channels by means of frequency hopping and time slicing. This allows extending the frequency diversity beyond the bandwidth of one RF channel and across hundreds of $\mathrm{MHz}$ (see Fig. 2). The utilization of TFS offers two potential benefits: improved reception due to better frequency diversity, and increased capacity due to more efficient statistical multiplexing. The TFS gain due to better frequency diversity is closely related to the frequency dependency of the transmitter site, the propagation path and the receivers [9].

Although TFS was originally proposed in the standardization process of DVB-T2, it was only included in the standard as an informative annex due to the need of implementing more than one tuner/front-end in receivers. Reception of TFS 
services with a single tuner requires a time interval between the slices of the same service in order to perform frequency hopping. Due to the high rates of HDTV services, the time interval needed for frequency hopping cannot be guaranteed in DVB-T2, and a second tuner is required for TFS operation. This is not a problem in DVB-NGH, as the lower bit rates of mobile TV services allow receivers to perform frame hopping with a single tuner.

\section{Multiple-Input Multiple-Output}

DVB-NGH distinguishes between rate 1 MIMO and rate 2 MIMO according to the multiplexing rate of information symbols across the transmit antennas. For rate 1 MIMO, DVB-NGH inherits the Alamouti code from DVB-T2 and also incorporates a novel technique known as enhanced single frequency network (eSFN). The main idea of eSFN is to apply a linear pre-distorsion function to each antenna in such a manner that the combination of the signals from different antennas results in a single transmit antenna channel with increased frequency diversity. The main advantage of eSFN is that better diversity is achieved without the need of additional pilot information or signal processing to demodulate the signal. Generally speaking, the number of pilots that must be transmitted for channel estimation purposes is proportional to the number of transmit antennas. This reduces the system capacity as a lower number of subcarriers in the OFDM symbols can be used for the transmission of data. While the Alamouti code requires doubling the amount of pilots that is transmitted for channel estimation, the number of pilots with eSFN is the same as with single transmit antenna systems.

For rate 2 MIMO, DVB-NGH has adopted a novel technique known as enhanced spatial multiplexing (eSM). This technique doubles the multiplexing rate of information symbols across the transmit antennas, and at the same time, achieves better robustness against spatial correlation. The presence of spatial correlation in the MIMO channel due to the lack of scattering or insufficient cross-polar discrimination (XPD) is specially detrimental for spatial multiplexing techniques [15]. In the case of eSM, the information symbols are weighted and combined before their transmission across the antennas to counter the presence of spatial correlation. In addition, a phase hopping term is added to the second antenna in order to randomize the code structure and avoid the negative effect of certain channel realizations.

\section{Performance Evaluation Based on the Outage CHANNEL CAPACITY}

\section{A. Formulation}

In this section, we describe the use of diversity from an information-theoretic point of view. To this purpose, we evaluate the performance of diversity techniques in mobile broadcasting systems based on the formulas that characterize the outage capacity of the channel. Under the assumption that the channel is unknown to the transmitter but perfectly known to the receiver, the channel capacity is given by [11]:

$$
C(\gamma)=\log _{2} \operatorname{det}\left(\mathbf{I}_{r}+\frac{\gamma}{t} \mathbf{H H}^{*}\right)
$$

where $\gamma$ is the carrier-to-noise ratio (CNR) and $\mathbf{H}$ is the $r \times t$ channel matrix with $r$ and $t$ being the number of receive and transmit antennas, respectively.

The presence of fading prevents error-free communication whenever the channel capacity in Eq. (1) drops below the transmission rate of information. In this case, the system is said to be in outage. The capacity of fading channels is generally expressed in outage terms as the maximum rate of information that can be achieved so that a target outage probability, $\epsilon$, is not exceeded [12]:

$$
C_{\epsilon}(\gamma)=\max \{R \mid p(\gamma, R) \leq \epsilon\},
$$

where $R$ is the rate of information and $p(\gamma, R)$ is the probability of being in outage:

$$
p(\gamma, R)=\operatorname{Pr}\{C(\gamma)<R\} .
$$

Mobile broadcasting networks are planned so that a certain QoS criterion, generally expressed in terms of outage probability, is satisfied in the coverage area. In order to compensate for fading and ensure that reliable communication is maintained at the target QoS, it is necessary to add an extra fading margin of several dBs to the link budget. Here, we define the fading margin gain $G_{\mathrm{F}}$ as the reduction in fading margin that can be achieved with diversity techniques. For the purpose of computing the fading margin gain, we also define the outage $\mathrm{CNR}$ as the minimum CNR that is required at a transmission rate $R$ so that a target outage probability $\epsilon$ is not exceeded:

$$
\gamma_{\epsilon}(R)=\min \{\gamma \mid p(\gamma, R) \leq \epsilon\} \text { bps } / \mathrm{Hz}
$$

After this, the fading margin gain achieved by any diversity technique can be easily computed as the difference between outage CNRs:

$$
G_{\mathrm{F}}(R)=\gamma_{\epsilon}^{\operatorname{div}}(R)-\gamma_{\epsilon}(R)
$$

For the purpose of computing the fading margin gains that can be achieved in mobile broadcasting systems with diversity techniques, we consider a system in which the FEC codewords are mapped on all the subcarriers in a given bandwidth, and across a number of OFDM symbols that is given by the interleaving duration. The capacity of such a system can be modelled as:

$$
C_{\text {broad }}(\gamma)=\frac{1}{L} \frac{1}{K} \sum_{l=1}^{L} \sum_{k=1}^{K} C_{l k}(\gamma) \mathrm{bps} / \mathrm{Hz}
$$

where $K$ is the total number of subcarriers covered by the transmission bandwidth, $L$ is the total number of OFDM symbols covered by the interleaving duration, and $C_{l k}$ is the channel capacity that correspond to the subcarrier $k$ of the OFDM symbol $l$. In this case, frequency diversity is determined by the transmission bandwidth (up to hundreds of $\mathrm{MHz}$ with TFS), time diversity is determined by the interleaving duration (up to several seconds with long TI), and space diversity is determined by the characteristics of the channel matrix $\mathbf{H}_{l k}$ in $C_{l k}$. 


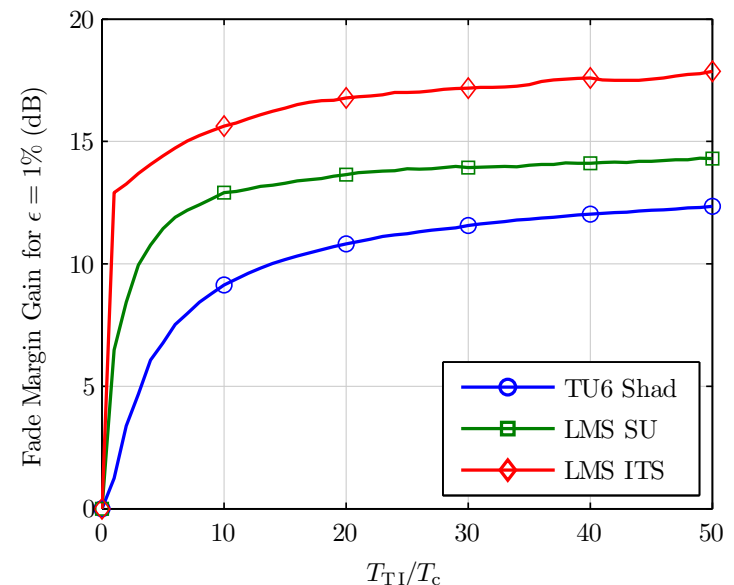

Fig. 3. Fading margin gains of long TI in terrestrial and satellite channels according to the number of coherence times covered by the interleaving duration.

\section{B. Channel Parameters}

The fading margin gains of diversity techniques have been computed in the 6-taps typical urban (TU6) channel model, which is representative of mobile reception in vehicular scenarios for a Doppler frequency $\left(f_{d}\right)$ above $10 \mathrm{~Hz}$. For the investigation on MIMO, the TU6 channel has been replaced by the NGH outdoor mobile channel [16]. This channel was developed during the standardization process of DVB-NGH, and is representative of UHF-band reception with crosspolarized antennas. The channel features a line-of-sight (LoS) component and a cross-polar discrimination (XPD) factor of $6 \mathrm{~dB}$. Additional terms for antenna rotation and asymmetry are also included. In particular, a rotation matrix describes the presence of polarization mismatch between the transmit and the receive antennas, whereas an asymmetry matrix describes the presence of power imbalance between different polarizations at the transmitter side.

For the investigation of long TI, we have considered the presence of shadowing in the TU6 and the NGH outdoor channels. In particular, we have assumed a user moving at constant velocity across a log-normal CNR map, which is defined by its standard deviation $(\sigma)$ and correlation distance $\left(d_{\text {corr }}\right)$ as described in [17]. In this case, the log-normal CNR map has been configured with $\sigma=5.5 \mathrm{~dB}$ and $d_{\text {corr }}=20 \mathrm{~m}$. In addition to terrestrial channels, we consider the reception in mobile satellite scenarios by using the statistical model for LMS presented in [18]. The model combines fast fading and shadowing by means of a Loo distribution with different parameters for each Markov state. The model is parameterized for different velocities $(v)$, elevation angles $(\theta)$, frequency bands, and reception environments. For our calculations, we have selected $v=60 \mathrm{~km} / \mathrm{h}, \theta=40$ degrees and $f_{\mathrm{RF}}=2.2$ $\mathrm{GHz}$ (S-band) in the satellite urban (SU) and intermediate tree shadowed (ITS) environments.

In the case of TFS, it is necessary to model the variations in the received signal strength between different RF channels in the UHF band. We assume that there is no correlation of fast fading between RF channels, whereas there is a certain correlation of shadowing. In addition to fast fading and shadowing, we consider the presence of a power imbalance caused, among other factors, by the frequency dependency of the antenna aperture and the height loss [19]. The correlation of shadowing in the frequency domain and the power imbalance between channels have been parameterized according to the statistical model presented in [9], which is based on field measurements performed by the Swedish terrestrial operator Teracom.

Unless stated otherwise, the results have been obtained for a target outage probability of $1 \%$ and a transmission rate of information of $2 \mathrm{bps} / \mathrm{Hz}$. In order to obtain statistical consistent results, the values presented in this chapter correspond to channel processes of several hours, which are difficult to obtain with physical layer simulations due to the required computational time.

\section{Time Diversity - Long TI}

In Fig. 3, we represent the fading margin gains of TI according to the number of coherence times ${ }^{1}$ that are covered by the interleaving duration. This allows us to assess the potential gain of TI regardless of the user velocity. The results show that TI can achieve gains as high as $18 \mathrm{~dB}$ in the LMS channels. In this case, the utilization of $\mathrm{TI}$ in the order of seconds is necessary to counter the presence of long signal outages caused by obstacles such as buildings and trees. It is important to notice that, although the largest gain is achieved in the LMS channels, gains up to $12 \mathrm{~dB}$ are also possible in the terrestrial scenario (TU6 channel with shadowing). According to the results, the gain of TI begins to saturate when the interleaving duration covers approximately fifty coherence times. In the case of shadowing with $d_{\text {corr }}=20 \mathrm{~m}$ and $v=60 \mathrm{~km} / \mathrm{h}$, fifty coherence times correspond with approximately $60 \mathrm{~s}$ of TI, which is generally not practical in mobile broadcasting systems. For a more realistic interleaving duration, such as $10 \mathrm{~s}$, the gain of TI is around $8 \mathrm{~dB}$ in the terrestrial scenario, and up to $15 \mathrm{~dB}$ in the LMS channels.

\section{Frequency Diversity - TFS}

Fig. 4 illustrates the fading margin gains of TFS according to the number of RF channels that constitute one TFS multiplex, and the separation between consecutive channels $\left(f_{s}\right)$. In this manner, the extent of interleaving in the frequency domain with $f_{s}=100 \mathrm{MHz}$ is around $100 \mathrm{MHz}$ for two channels, $200 \mathrm{MHz}$ for three channels, and so on. The larger the extent of interleaving in the frequency domain, the better the protection achieved against the signal variations between channels. It should be noted, however, that some configurations shown in Fig. 4 are not practical in real scenarios, since they exceed the bandwidth assigned to DTT services in the UHF band $(\leq 400 \mathrm{MHz})$. For a configuration using four RF channels and $f_{s}=100 \mathrm{MHz}$, the gain of TFS is around $7 \mathrm{~dB}$. This would be the case of a TFS multiplex comprising the RF channels located in $470 \mathrm{MHz}, 570 \mathrm{MHz}, 670 \mathrm{MHz}$ and $770 \mathrm{MHz}$.

\footnotetext{
${ }^{1}$ The coherence time, $T_{c}$, is defined as the period of time over which the propagation channel remains constant. In the case of shadowing, it can be approximated by $T_{c} \approx d_{\mathrm{corr}} / v$, where $v$ is the user velocity and $d_{\mathrm{corr}}$ is the correlation distance.
} 


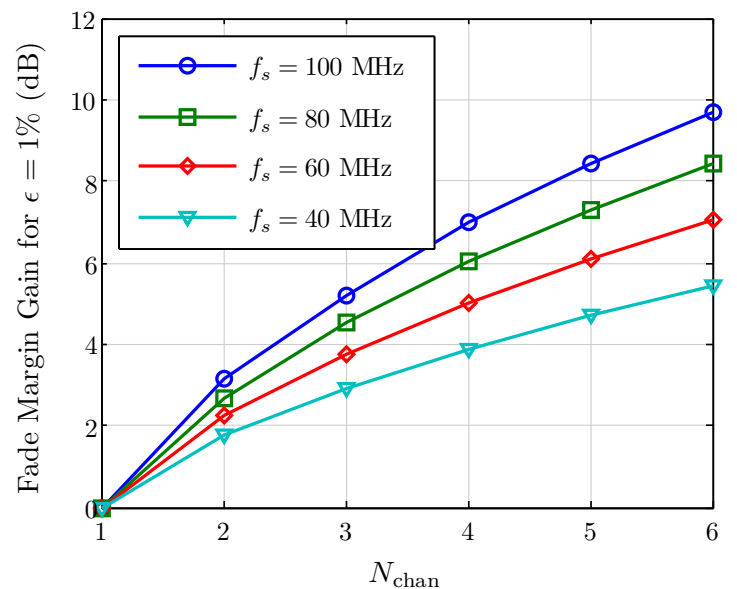

Fig. 4. Fading margin gain of TFS in the TU6 channel with shadowing according to the number of RF channels that constitute one TFS multiplex and the separation between consecutive channels $\left(f_{s}\right)$.

\section{E. Space Diversity - Cross-polar MIMO}

In Fig. 5 we represent the fading margin gains of crosspolar MIMO. The results show that the use of cross-polarized antennas for MIMO purposes can achieve important gains in mobile broadcasting systems. In this case, cross-polarized antennas provide better robustness against polarization impairments such as rotation and asymmetry, even if the presence of correlation between antenna paths reduces the diversity gain against fading. The only exception to this is MISO, for which the use of multiple antennas results in a performance loss compared to SISO. This is explained by the fact that, with cross-polarized antennas, half of the transmission power that is assigned to the orthogonal polarization is lost when there is only one antenna at the receiver side. As a result, the utilization of cross-polar MISO in mobile broadcasting systems has very limited applicability.

In Fig. 5, we can also see that the gain of rate 2 MIMO grows linearly with the rate of information, whereas the gain for the rest of configurations remains constant. In this sense, it is important to distinguish between the diversity gain that results from better protection against fading, and the multiplexing gain that is achieved by increasing the transmission rate of information symbols across the antennas. The first does not depends on the CNR and can be seen as an offset in the outage capacity of the channel, whereas the second modifies the rate of growth of the capacity with the CNR. As a result, the advantage of rate 2 MIMO over rate 1 MIMO grows when the rate of information increases. For $1 \mathrm{bps} / \mathrm{Hz}$, which is a transmission rate characteristic of handheld reception (i.e. QPSK code rate 1/2), the fading margin gains of rate 2 MIMO and rate $1 \mathrm{MIMO}$ are similar. With $4 \mathrm{bps} / \mathrm{Hz}$, which is more typical of fixed and vehicular reception (e.g 64QAM code rate $2 / 3$ ), rate 2 MIMO outperforms rate 1 MIMO in about $3 \mathrm{~dB}$.

\section{F. Combined Time, Frequency and Space Diversity}

The influence of TFS in the performance of long TI can be seen in Fig. 6, where we represent the fading margin

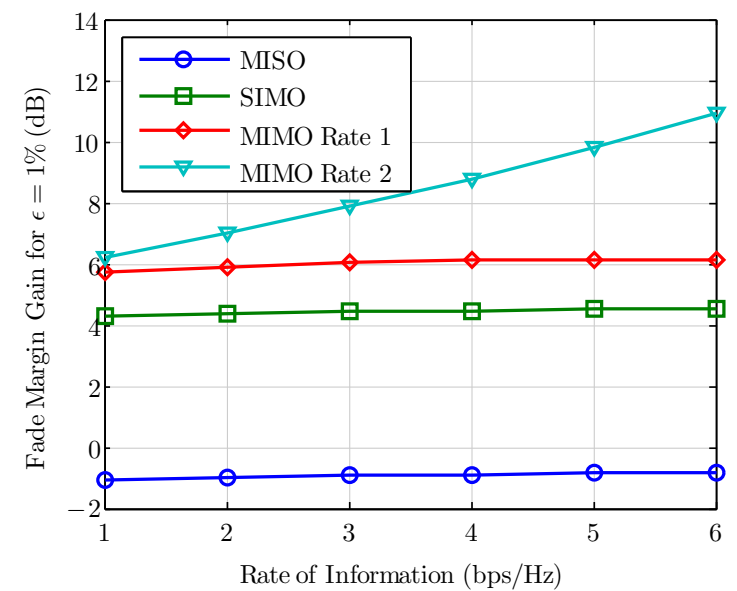

Fig. 5. Fading margin gains of cross-polar MIMO in the NGH outdoor channel according to the transmission rate of information.

gains of long TI for different configurations of TFS with $f_{s}=100 \mathrm{MHz}$. According to the results, the utilization of TFS can reduce the gain achieved by long TI in several dBs. The reason is that TFS can compensate for shadowing along the user trajectory by means of improved frequency diversity, and therefore, it lowers the benefits of using long TI. This is in accordance with the diminishing marginal returns of diversity: the higher the performance gain achieved with TFS, the lower the gain achieved with long TI and vice versa.

In Fig. 7, we represent the fading margin gains of long TI with different antenna configurations in the NGH outdoor channel. We can see that, compared to TFS, the utilization of cross-polar MIMO has very little impact in the performance of long TI. This is explained by the fact that the gain of TI comes from better protection against fast fading and shadowing, whereas the gain of cross-polar MIMO is mostly due to better robustness against rotation and asymmetry. Consequently, the performance of TI and MIMO does not depend on each other, and the gain of combining both techniques is approximately equal to the sum of the individual gains. We can also see that the gain of TI for long interleaving durations is approximately $0.5 \mathrm{~dB}$ higher in the case of rate $2 \mathrm{MIMO}$ than for the rest of configurations. The reason is that long TI is beneficial for the multiplexing gain of MIMO techniques, a this depends on the average CNR encountered along the interleaving duration.

\section{Simulation Results}

In addition to the investigation based on channel capacity, we have studied the use of diversity in DVB-NGH systems by means of physical layer simulations. The simulator has been calibrated under the standardization process of DVB-NGH. Together with the simulation results, we represent the outage probability of the channel according to Eq. (3) in order to compare the performance of DVB-NGH with the outage channel capacity. This has been computed for the same interleaving duration and Doppler frequency than the physical layer simulations. 


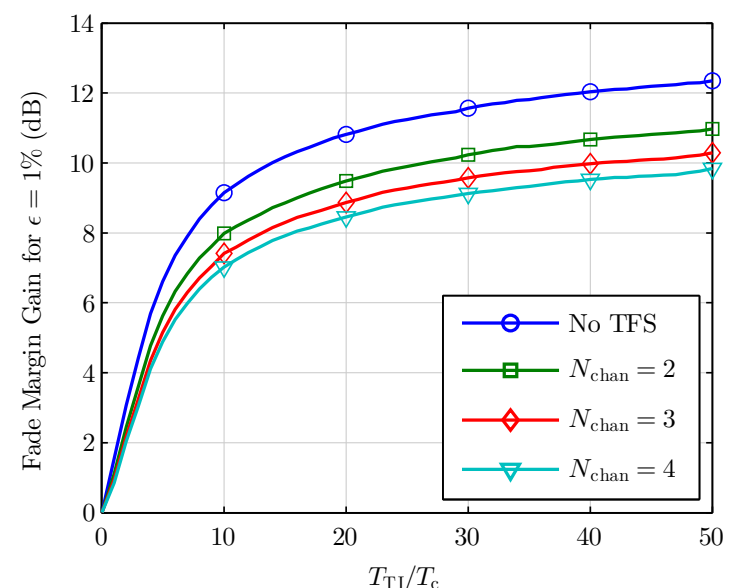

Fig. 6. Fading margin gain of long TI in the TU6 channel with shadowing for different configurations of TFS ( $f_{s}=100 \mathrm{MHz}$ ), and according to the number of coherence times covered by the interleaving duration.

\section{A. Long $T I$}

In Fig. 8, we can see the performance of DVB-NGH with different configurations of TI. In the left side of the figure, the results show the BBFER curves corresponding to intraframe $\mathrm{BI}\left(T_{\mathrm{TI}}=200 \mathrm{~ms}\right)$ and inter-frame CI $\left(T_{\mathrm{TI}}=10 \mathrm{~s}\right)$ with uniform and uniform-late configurations. The uniformlate configurations selected for the simulations carry a $50 \%$, $40 \%$ and $30 \%$ of information in the late frame, and are refered to as UL 50\%, UL $40 \%$ and UL $30 \%$ respectively. The outage probability of the channel for $200 \mathrm{~ms}$ and $10 \mathrm{~s}$ of TI is also shown as reference.

From the results, we can see that the use of uniform-late interleaving reduces the gain of TI. This is in accordance to the trade-off between zapping performance and time diversity that exists in DVB-NGH: uniform-late interleaving lowers the zapping time but degrades the reception robustness as a result of worse time diversity. The greater the percentage of information carried in the late part, the higher the degradation. In the case of QPSK and code rate 1/3, the performance loss of the uniform-late interleaving compared to uniform interleaving is around $0.5 \mathrm{~dB}, 1 \mathrm{~dB}$ and $2 \mathrm{~dB}$ for UL $30 \%$, UL $40 \%$ and UL $50 \%$ respectively. In the figure, we can also see that the performance gap between DVB-NGH and the outage capacity of the channel is larger with inter-frame CI than with intra-frame BI. This is explained by the fact that, with intra-frame BI, the interleaving duration is much shorter than the coherence time of the channel, and therefore, the presence of shadowing results in an offset of the average CNR in each codeword. With inter-frame CI, the interleaving duration covers several coherence times, and in this case, the gain of TI depends on the error-correction capabilities of the FEC code against shadowing.

In the right side of Fig. 8, the results show the gain of long TI with inter-frame CI compared to intra-frame BI for code rates between $1 / 3$ and $11 / 15$. The dashed line has been computed according to the outage capacity of the channel, and represents the highest gain that can be achieved with ideal FEC

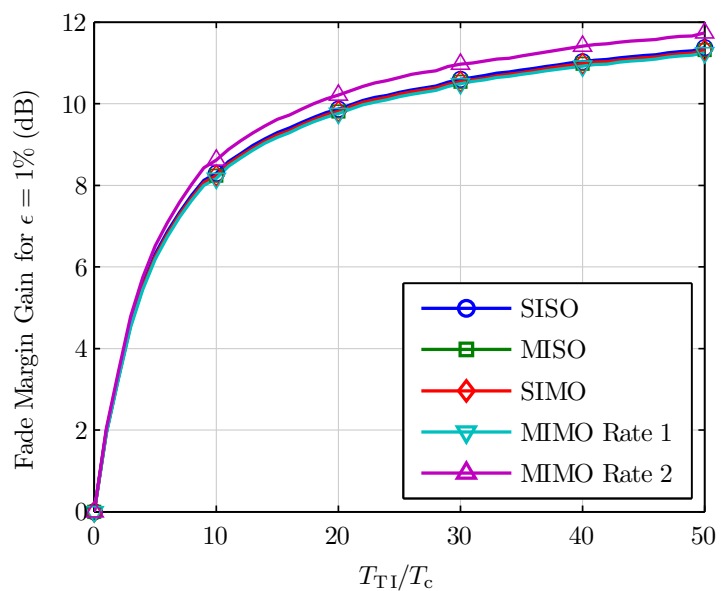

Fig. 7. Fading margin gain of long TI in the NGH outdoor channel with shadowing for different configurations of cross-polar MIMO, and according to the number of coherence times covered by the interleaving duration.

and interleaving. Due to the fact that it is based on Shannon limit, the gain of long TI in this case does not depend on particular aspects of bit-interleaved coded modulation (BICM) systems such as the constellation or the code rate. On the contrary, the gain of long TI in DVB-NGH diminishes with higher code rates, since it depends on the error-correcting capabilities of the FEC code. This means that in order to take full advantage of long TI and approach the outage capacity of the channel, it is necessary to employ low code rates as well as uniform interleaving.

\section{B. TFS}

Fig. 9 illustrates the performance of DVB-NGH with TFS according to the number of RF channels that constitute the TFS multiplex. The results have been obtained for $f_{s}=100 \mathrm{MHz}$ and a maximum of four channels. As can be seen in the figure, the achievable gain of TFS in DVB-NGH is consistent with the information-theoretic results presented in Fig. 4. We can also see that the performance gap between DVB-NGH and the outage capacity grows larger with the number of RF channels in the TFS multiplex. This is similar to the case of long TI in the sense that, while the utilization of extended interleaving improves the reception robustness as a result of better diversity, the gain depends on the error-correcting capabilities of the FEC code (i.e. code rate).

In the right side of Fig. 9, we can see more clearly the influence of the code rate in the performance gains that can be achieved with TFS. The results show two important aspects regarding the performance of TFS in BICM systems, and particularly, DVB-NGH. On one hand, the distance to the outage capacity of the channel increases with the number of channels, since it is necessary to cope with a larger signal variability in the frequency domain. On the other hand, the gain of TFS diminishes with higher code rates. As can be seen in Fig. 9, the higher the number of channels, the greater the dependency of the system performance on the code rate. 

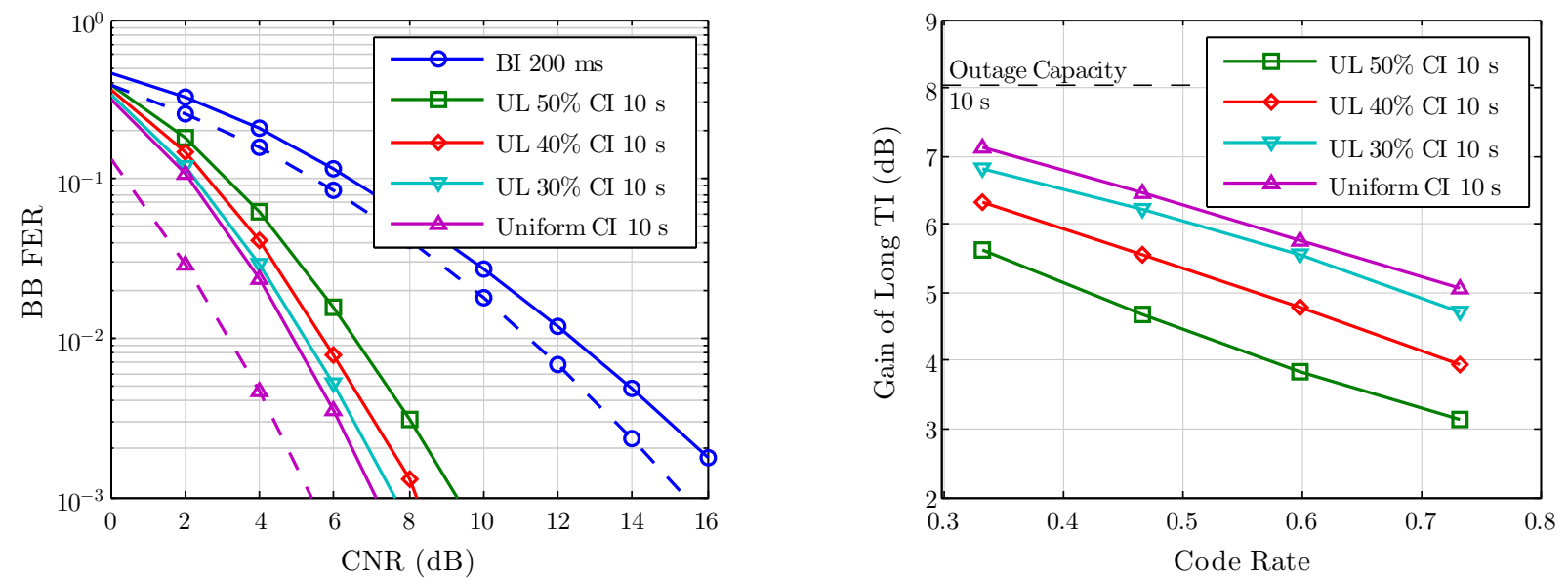

Fig. 8. Performance of DVB-NGH with different configurations of TI in the TU6 channel with shadowing $\left(f_{d}=33 \mathrm{~Hz}, \sigma=5.5 \mathrm{~dB}, d_{\mathrm{corr}}=20 \mathrm{~m}\right)$. On the left, BBFER curves with QPSK and code rate $1 / 3$. On the right, gain of inter-frame CI ( $\left.T_{\mathrm{TI}}=10 \mathrm{~s}\right)$ compared to intra-frame BI $\left(T_{\mathrm{TI}}=200 \mathrm{~ms}\right)$ with QPSK and different code rates. Dashed lines correspond to the outage probability of the channel (left), and to the gain of long TI according to the outage capacity of the channel (right).
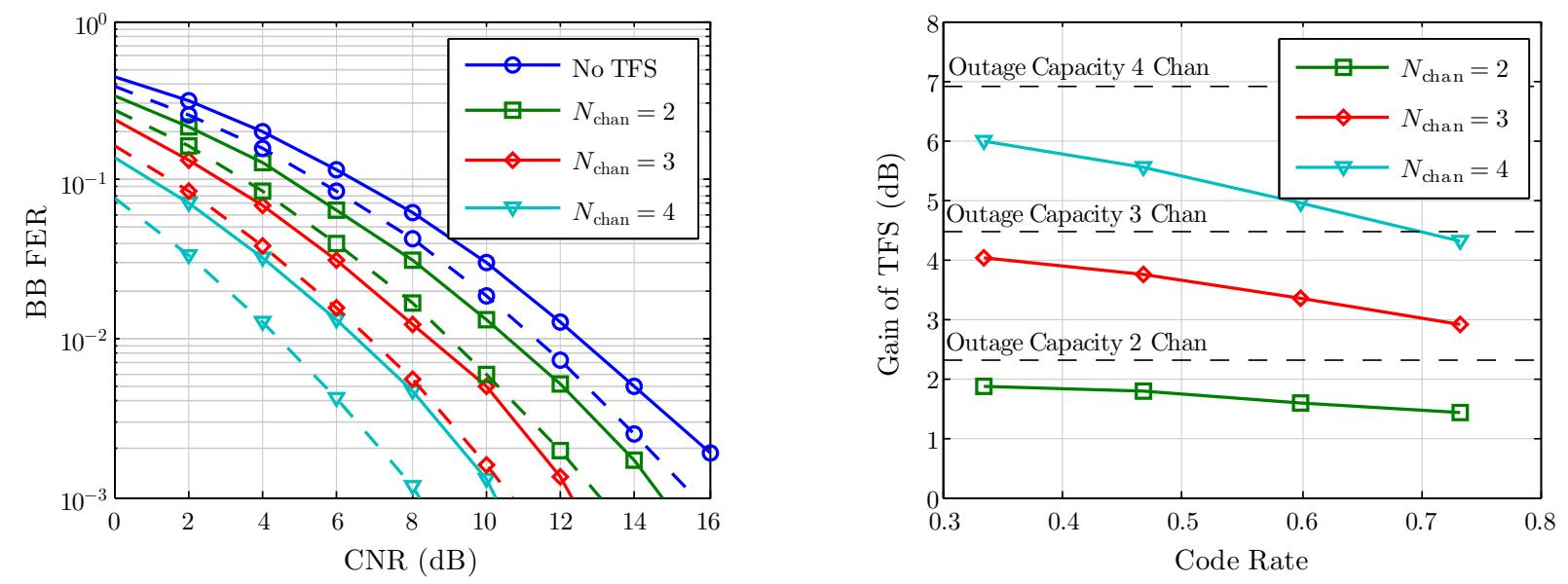

Fig. 9. Performance of DVB-NGH with TFS in the TU6 channel with shadowing $\left(f_{d}=33 \mathrm{~Hz}, \sigma=5.5 \mathrm{~dB}, d_{\text {corr }}=20 \mathrm{~m}, f_{s}=100 \mathrm{MHz}\right)$. On the left, BBFER curves with QPSK and code rate 1/3. On the right, gain of TFS with QPSK and different code rates. Dashed lines correspond to the outage probability of the channel (left), and to the gain of TFS according to the outage capacity of the channel (right).

\section{Cross-polar MIMO}

Fig. 9, we can see the performance of DVB-NGH with the different MIMO techniques that have been included in the standard. The BBFER curves corresponding to SISO, SIMO, eSFN and the Alamouti code with QPSK and code rate 1/3 are shown in the left side of the figure. The curve corresponding to eSM is not represented since it is only supported for constellations higher than 16QAM. The simulation results show that the gain of MIMO techniques in DVB-NGH can be as high as $6 \mathrm{~dB}$ at low CNR values, which is line with the information-theoretic results presented in Fig. 5. We can see that the Alamouti code achieves the largest gain followed by eSFN and SIMO. As an optimal rate 1 MIMO scheme, the Alamouti code exploits the full diversity of the MIMO channel, and therefore, it provides better reception robustness than a suboptimal technique from the point of view of diversity such as eSFN. Nevertheless, it is important to notice that, although eSFN is outperformed by the Alamouti code, the performance gap between both schemes is less than $0.5 \mathrm{~dB}$.

In the right side of Fig. 10, we represent the capacity curves of DVB-NGH for the MIMO schemes adopted in the standard, including eSM. The transmission of pilots for channel estimation purposes has been taken into account. In particular, we have assumed the same considerations as in the DVB-NGH standardization process, i.e. pilot overhead 1/12 for SISO, SIMO, and eSFN, and pilot overhead 1/6 for the Alamouti code and eSM. This corresponds to a robust configuration that provides sufficient quality of channel estimation in mobile scenarios. In this case, eSFN outperforms the Alamouti code across the entire range of $\mathrm{CNR}$ values, as the better reception robustness achieved by the Alamouti code does not compensate for the higher pilot overhead in terms of spectral efficiency. We can also see that eSM achieves the best performance in the high CNR region due to the multiplexing 

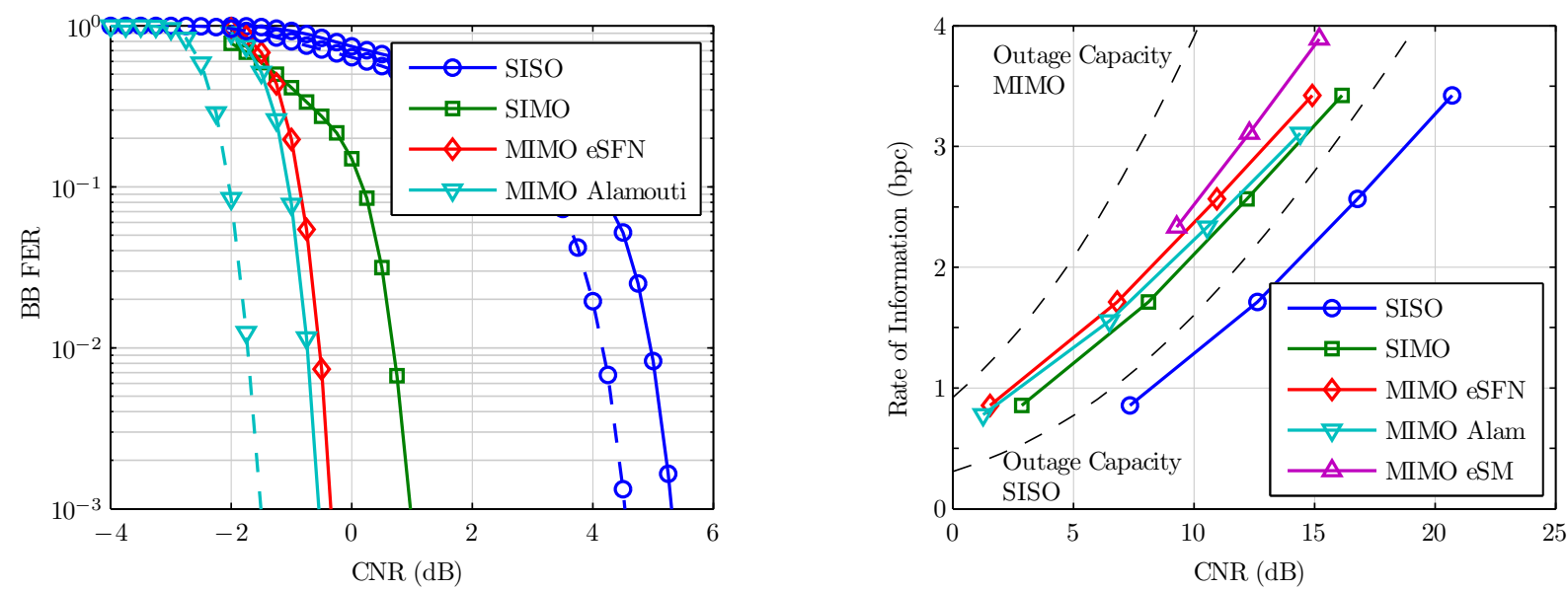

Fig. 10. Performance of DVB-NGH with cross-polar MIMO in the NGH outdoor channel $\left(f_{d}=33 \mathrm{~Hz}\right)$. On the left, BBFER curves with QPSK and code rate $1 / 3$. On the right, capacity curves with code rate $7 / 15$ and different constellations. Dashed lines correspond to the outage probability of the channel (left), and to the outage capacity of the channel (right).

gain of rate 2 MIMO schemes. In this case, the utilization of eSM is especially interesting to achieve higher spectral efficiencies than supported by rate 1 MIMO configurations. For example, the spectral efficiency with eSM at $15 \mathrm{~dB}$ of CNR is around 4 bits per cell (bpc), which represents a capacity increase in the order of $15 \%$ compared to eSFN. On the other hand, the performance gap between DVB-NGH and the outage capacity of the channel is significantly larger with MIMO than with SISO. These results highlight the need to implement iterative decoding and demapping techniques in order to fully exploit the multiplexing capabilities of the MIMO channel in the high CNR region [20].

\section{Combined Diversity}

In the previous sections of this chapter, we have evaluated in a separate manner the performance gains of time, frequency and space diversity in DVB-NGH. In this section, we focus on the combination of different types of diversity, and particularly, on the performance gains that can be achieved by combining long TI with TFS and cross-polar MIMO. The results in the left side of Fig. 11 correspond to the combination of long TI with TFS. As predicted by the results based on the outage channel capacity in Fig. 6, the total gain of combining both techniques is lower that the sum of the individual gains due to the diminishing marginal returns of diversity. In this case, the gain of long TI with $T_{\mathrm{TI}}=10 \mathrm{~s}$ is $1 \mathrm{~dB}$ lower when the transmission extends across four RF channels instead of one RF channel. Similarly, the gain of TFS with four channels is $1 \mathrm{~dB}$ lower when the TI extends over $10 \mathrm{~s}$ with inter-frame CI rather than $200 \mathrm{~ms}$ with intra-frame BI.

In the right side of Fig. 11, we show the results corresponding to the combination of long TI with MIMO. According to the figure, the total gain of combining both techniques is approximately equal to the sum of the individual gains. This is consistent with the results based on the outage channel capacity in Fig. 7. It is important to note that in the case of cross-polarized antennas, the majority of the gain does not come from fading, but from better protection against rotation and asymmetry effects. These are considered to persist over periods of time much longer than the interleaving duration, and therefore, cannot be compensated by means of TI.

\section{Discussion on Network Planning}

The advantage of diversity techniques in terms of network planning is twofold: on one hand, the fading margin gains can be interpreted as a coverage extension of mobile services, or alternatively, as a reduction of the network infrastructure that is required to provide acceptable coverage levels. On the other hand, they can be translated into a higher capacity for the transmission of mobile services by means of higher code rates and less robust constellations. This allows accommodating more programs in the same bandwidth, or using a higher bitrate per channel to elevate the audio-visual quality of services.

The fading margin gains of TFS and MIMO can be incorporated directly into the link margin, and therefore, it is possible to estimate in a simple manner the coverage extension that is achieved in DVB-NGH networks. Using the methodology presented in [6], we have first computed the relative increase in the cell radius for a distance dependent propagation exponent of 3.5 (typical urban environment). Assuming a cell of circular shape, the gain in coverage area is equal to the square of the cell radius gain.

In Table I, we show the fading margin gains and the corresponding coverage extension for different configurations of TFS and MIMO in DVB-NGH. According to the values listed in the table, the utilization of TFS with four channels and rate 1 MIMO can multiply by a factor higher than two the coverage area of mobile services. If both techniques are combined, the coverage area can be extended almost by a factor of five. It should be pointed out that, contrary to the case of TFS and MIMO, the fading margin gains of long TI cannot be translated directly into a coverage extension of mobile services due to the fact that they depend on the 

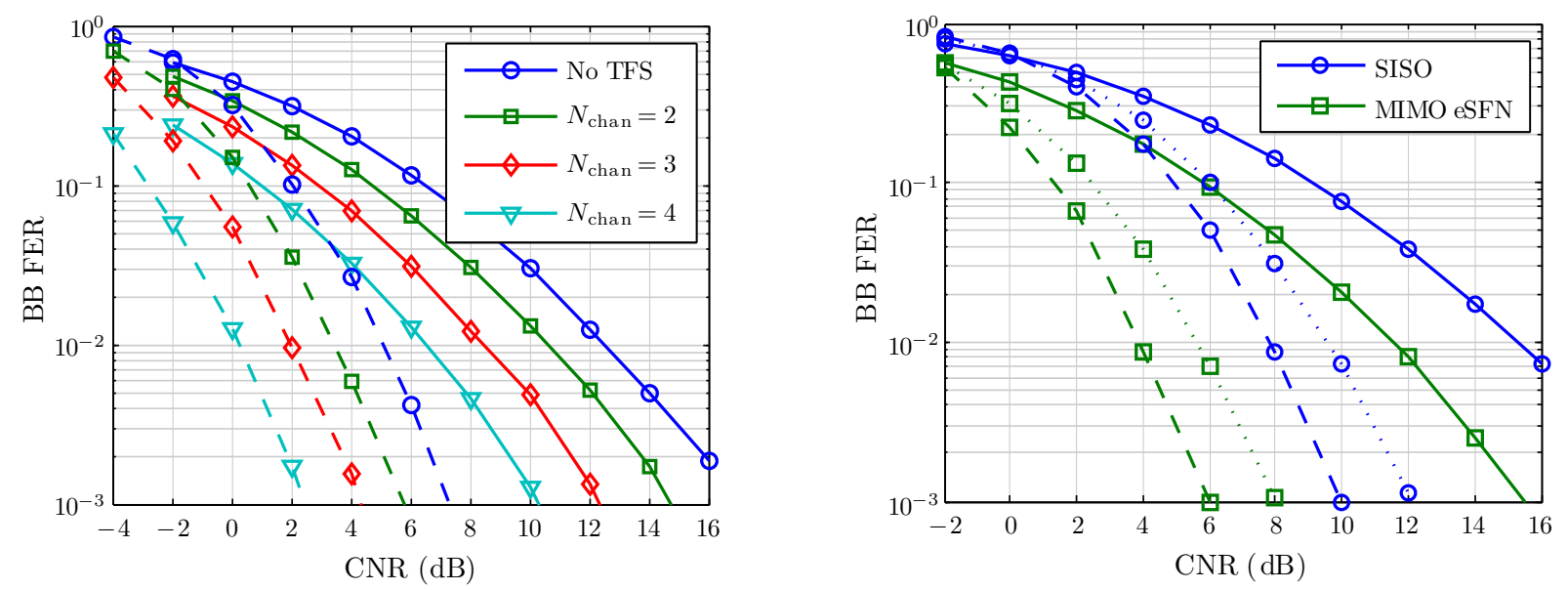

Fig. 11. Combined diversity in DVB-NGH systems (QPSK 1/3). On the left, long TI with TFS in the TU6 channel with shadowing $\left(f_{d}=33 \mathrm{~Hz}\right.$, $\sigma=5.5 \mathrm{~dB}, d_{\text {corr }}=20 \mathrm{~m}, f_{s}=100 \mathrm{MHz}$ ). On the right, long TI with cross-polar MIMO (eSFN) in the NGH outdoor channel with shadowing. Continuous lines correspond to intra-frame BI $\left(T_{\mathrm{TI}}=200 \mathrm{~ms}\right)$, dashed lines correspond to uniform inter-frame CI ( $\left.T_{\mathrm{TI}}=10 \mathrm{~s}\right)$, and dotted lines correspond to UL $50 \%$ inter-frame $\mathrm{CI}\left(T_{\mathrm{TI}}=10 \mathrm{~s}\right)$

TABLE I

FADING MARGIN GAINS AND COVERAGE EXTENSION

\begin{tabular}{|c|c|c|c|}
\hline & & Fading margin gain & Coverage extension \\
\hline \multirow{3}{*}{ TFS } & 2 channels & $2 \mathrm{~dB}$ & $30 \%$ \\
\hline & 3 channels & $4 \mathrm{~dB}$ & $70 \%$ \\
\hline & 4 channels & $6 \mathrm{~dB}$ & $120 \%$ \\
\hline \multirow[t]{2}{*}{ MIMO } & SIMO & $4 \mathrm{~dB}$ & $70 \%$ \\
\hline & MIMO rate 1 & $6 \mathrm{~dB}$ & $120 \%$ \\
\hline \multicolumn{2}{|c|}{$\begin{array}{l}\text { TFS with } 4 \text { channels } \\
+ \text { MIMO rate } 1\end{array}$} & $12 \mathrm{~dB}$ & $385 \%$ \\
\hline
\end{tabular}

velocity and trajectory of the users. For example, the gain of TI with moderate to long interleaving durations is considerable smaller if the user remains stationary than if it is moving across the service area. Therefore, it is necessary to perform dynamic system level simulations that take into account the user mobility in order to quantify the network gain of long TI [21].

The capacity gains of diversity techniques refer to the possibility of using less robust configurations of constellation and code rate, while ensuring the same reception robustness and coverage area. In real systems, however, the capacity gains are limited by the range of constellations and code rates that can be selected for transmission. For example, it is not always possible to trade a certain fading margin gain for higher capacity, if the standard does not include the combination of constellation and code rate that can provide the same robustness, but with better spectral efficiency. In order to evaluate the capacity gains of diversity techniques from a more generic point of view, we employ a methodology based on the outage capacity of the channel. In particular, we compute the capacity gain of diversity techniques, $G_{\mathrm{C}}$, as the relative increase in terms of spectral efficiency:

$$
G_{\mathrm{C}}(\gamma)=\frac{C\left(\gamma_{\epsilon}-G_{F}\right)-C\left(\gamma_{\epsilon}\right)}{C\left(\gamma_{\epsilon}\right)}
$$

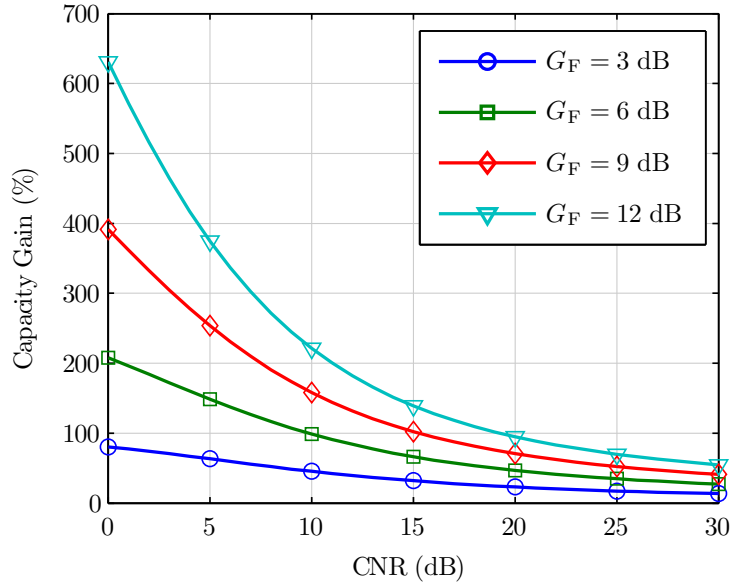

Fig. 12. Correspondence between fading margin gain and capacity gain according to the rate of information in the NGH outdoor channel.

The curves in Fig. 12 show the capacity gain corresponding to fading margin gains of $3 \mathrm{~dB}, 6 \mathrm{~dB}, 9 \mathrm{~dB}$ and $12 \mathrm{~dB}$ in the NGH outdoor channel. We can see that, for any given fading margin gain, the capacity gain that can be achieved by using a less robust configuration of constellation and code rate diminishes with the CNR. It is remarkable that in the low CNR region, the utilization of rate 1 MIMO and TFS $\left(G_{\mathrm{F}}=6 \mathrm{~dB}\right)$ can triple $\left(G_{\mathrm{C}} \approx 200 \%\right)$ the capacity of mobile services, while the combination of both techniques $\left(G_{\mathrm{F}}=12 \mathrm{~dB}\right)$ can multiply the capacity by a factor of seven $\left(G_{\mathrm{C}} \approx 600 \%\right)$.

\section{Conclusions}

DVB-NGH is the first broadcasting system to exploit the diversity in the time, frequency and space domains by incorporating at the physical layer long TI, TFS and cross-polar MIMO. In this paper, we have investigated the use of diversity in the three domains from an information-theoretic point of 
view based on the outage capacity of the channel, as well as with physical layer simulations.

The results based on the outage capacity of the channel reveal that the potential gain of TI in LMS and terrestrial scenarios is very high, provided that the interleaving duration is long enough. For interleaving durations in the order of $10 \mathrm{~s}$, the gain of TI is around $15 \mathrm{~dB}$ in LMS channels, and around $8 \mathrm{~dB}$ in terrestrial scenarios. However, these gains are conditioned by the user velocity and trajectory during the interleaving duration, and therefore, they cannot be translated directly into a coverage extension of mobile services. Similarly to long TI, the incorporation of TFS has the potential to achieve very large gains in mobile broadcasting systems. In this case, the gain is determined by the extent of interleaving in the frequency domain. For a configuration of TFS with four $\mathrm{RF}$ channels along $300 \mathrm{MHz}$, it is possible to improve the reception robustness in up to $7 \mathrm{~dB}$. The information-theoretic results also reveal that SIMO and MIMO configurations based on cross-polarized antennas can achieve important gains in mobile broadcasting systems as a result of better protection against polarization impairments. Rate 1 MIMO schemes (diversity) can achieve gains in the order of $6 \mathrm{~dB}$, whereas the gain of rate 2 MIMO schemes (diversity and multiplexing) grows larger in the high CNR region. In contrast, the utilization of cross-polar MISO has very little applicability due to the lack of an uplink channel in this kind of systems.

The physical layer simulations are in line with the information-theoretic results. In the case of time diversity, the simulations show that in order to take full advantage of long TI in DVB-NGH and approach the outage capacity of the channel, it is necessary to employ low code rates as well as uniform interleaving. In this sense, uniform-late configurations of interleaving can reduce the zapping time at the expense of worse time diversity. Low code rates are also needed in order to approach the outage capacity of the channel in the case of TFS. The simulations show that the larger the extent of interleaving in the frequency domain, the greater the dependency of the system performance on the code rate. In the case of MIMO, the amount of capacity devoted to the transmission of pilots has a significant impact in the performance of MIMO schemes. According to the simulation results, a sub-optimal scheme from the point of view of diversity such as eSFN can outperform optimal techniques like the Alamouti code in terms of spectral efficiency.

Regarding the combined use of diversity, both the information-theoretic results and the physical layer simulations reveal that while the combination of long TI and TFS can achieve very large gains in realistic scenarios, the total gain of combining both techniques is lower than the sum of the individual gains. The reason is that both techniques improve the protection against shadowing, and therefore, the utilization of one technique diminishes the gain that can be achieved with the other. In contrast, the influence of MIMO in the performance gain of long TI is much more limited due to the fact that most of the MIMO gain with cross-polarized antennas comes from rotation and asymmetry. Consequently, the combined gain of long TI and cross-polar MIMO is approximately equal to the sum of the individual gains.
In summary, the results presented in this paper demonstrate that the combined use of diversity in the time, frequency and space domains is fundamental to satisfy the commercial requirements of next generation mobile broadcasting systems, which demand more efficient network infrastructure and enhanced robustness.

\section{REFERENCES}

[1] F. Selgert, "Commercial requirements for DVB-NGH," DVB Document CM-NGH015R1, 2009

[2] M. Eroz, L. N. Lee, and F. W. Sun, "Impact of near shannon-limit codes on wireless communications technology," in IEEE International Symposium on Personal, Indoor and Mobile Radio Communications (PIMRC'04), Barcelona, Spain, Sep. 2004.

[3] G. Faria, J. A. Henriksson, E. Stare, and P. Talmola, "DVB-H: Digital broadcast services to handheld devices," Proceedings of the IEEE, vol. 94, no. 1, pp. 194-209, 2006.

[4] P. Kelley and C. Rigal, "DVB-SH - Mobile digital TV in S band," Technical Review, EBU, 2007.

[5] J. Lei, M. A. Vázquez-Castro, and T. Strockhammer, "Link layer FEC and cross-layer architecture for DVB-S2 transmission with QoS in railway scenarios," IEEE Transactions on Vehicular Technology, vol. 58, no. 8, pp. 4265-4276, 2009.

[6] D. Gómez-Barquero, D. Gozálvez, and N. Cardona, "Application layer FEC for mobile TV delivery in IP datacast over DVB-H systems," IEEE Transactions on Broadcasting, vol. 55, no. 2, pp. 396-406, 2009.

[7] Upper Layer Forward Error Correction for DVB Systems (ULFEC), ETSI Std. TS 102 993, Rev. 1.1.1, 2011.

[8] M. Makni, J. Robert, and E. Stare, "Performance analysis of time frequency slicing," in ITG Conference on Electronic Media Technology (CEMT'11), Dortmund, Germany, Mar. 2011.

[9] J. J. Giménez, D. Gozálvez, D. Gómez-Barquero, and N. Cardona, “A statistical model of the signal strength imbalance between RF channels in a DTT network," Electronics Letters, pp. 731-732, 2012.

[10] C. Gómez-Calero, L. C. Navarrete, L. de Haro, and R. Martínez, "A 2x2 MIMO DVB-T2 system: Design, new channel estimation scheme and measurements with polarization diversity," IEEE Transactions on Broadcasting, vol. 56, no. 2, pp. 184-192, 2010.

[11] L. Zheng and D. N. C. Tse, "Diversity and multiplexing: A fundamental tradeoff in multiple-antenna channels," IEEE Transactions on Information Theory, vol. 49, no. 5, pp. 1073-1096, 2003.

[12] L. H. Ozarow, S. Shamai, and A. D. Wyner, "Information theoretic considerations for cellular mobile radio," IEEE Transactions on Vehicular Technology, vol. 43, no. 2, pp. 359-378, 1994.

[13] E. Telatar, "Capacity of multi-antenna gaussian channels," European Transactions on Telecommunications, vol. 10, no. 6, pp. 585-596, 1999.

[14] Guidelines for Implementation for Satellite Services to Handheld devices (SH) below 3GHz, ETSI Std. TS 102 584, Rev. 1.2.1, 2011.

[15] R. U. Nabar, H. Bolcskei, and A. J. Paulraj, "Transmit optimization for spatial multiplexing in the presence of spatial fading correlation," in IEEE Global Telecommunications Conference (GLOBECOM'01), San Antonio, USA, Nov. 2001.

[16] P. Moss, T. Y. Poon, and J. Boyer, "A simple model of the UHF crosspolar terrestrial channel for DVB-NGH," White Paper, BBC, 2011.

[17] S. Saunders and A. Aragón-Zabala, Antennas and Propagation for Wireless Communication Systems, 2nd ed. Wiley, 2007.

[18] F. P. Fontán, M. Vázquez-Castro, C. E. Cabado, J. P. Garca, and E. Kubista, "Statistical modeling of the LMS channel," IEEE Transactions on Vehicular Technology, vol. 50, no. 6, pp. 1549-1567, 2001.

[19] "Frequency and network planning aspects of DVB-T2," Technical Report, EBU Tech 3348, 2012.

[20] B. M. Hochwald and S. Brink, "Achieving near-capacity on a multipleantenna channel," IEEE Transactions on Communications, vol. 51, no. 3 , pp. 389-399, 2003.

[21] D. Gómez-Barquero, P. Unger, T. Kurner, and N. Cardona, "Coverage estimation for multiburst FEC mobile TV services in DVB-H systems," IEEE Transactions on Vehicular Technology, vol. 59, no. 7, pp. 34913500, 2010. 